\title{
A Cognitive Perspective on the Safety Communication Factors That Affect Worker Behavior
}

\author{
Pinchao Liao', Liuxin Jiang1, Bingsheng Liu ${ }^{2 *}$, Changtao Chen1, Dongping Fang1, \\ Peilun Rao3, Mengchun Zhang1 \\ ${ }^{1}$ Department of Construction Management, Tsinghua University, Beijing, China \\ ${ }^{2}$ School of Management and Economics, Tianjin University, Tianjin, China \\ ${ }^{3}$ Department of Industrial Engineering, Tsinghua University, Beijing, China \\ Email: ${ }^{*}$ bluesea boy 1979@163.com
}

Received 28 May 2014; revised 1 August 2014; accepted 26 August 2014

Copyright (C) 2014 by authors and Scientific Research Publishing Inc.

This work is licensed under the Creative Commons Attribution International License (CC BY). http://creativecommons.org/licenses/by/4.0/

(c) (i) Open Access

\begin{abstract}
Communication is vital for construction safety, but how it influences unsafe behavior in the workplace remains unclear. This study aims to explore the relationships between communication, cognitive failure, and unsafe behaviors in order to clarify those mechanics. We defined communication as management input, worker cognitive failure as process, and actual behaviors as management output. We collected data from three Chinese steel construction crews and utilized experience/behavior sampling and questionnaire surveys to collect project information. Bivariate statistical analyses were conducted to explain how communication affected unsafe behavior. The results showed that, rather than communication frequency, management communication style was significantly related to worker cognitive failure; specifically, communication style was related to perception of convenience and self-capacity, which could be upstream factors explaining unsafe behavior at the construction jobsite. This research provided statistical evidence supporting the hypothesized association among safety communication, cognitive failure, and behavior, bridging the missing gaps of previous research. Nonetheless, readers should interpret the results cautiously because of the limitations listed as follows. First, sample size is relatively small. In addition, crew turnover may mediate the relationship between communication and safe behaviors therefore, the impact of communication on cognitive failure can be blurred and a longitudinal study on specific crews working on the same job site could be used to evaluate it. Third, future research should focus on communication style, as it directly relates to the human cognitions that affect unsafe behavior at construction sites.
\end{abstract}

${ }^{*}$ Corresponding author.

How to cite this paper: Liao, P.C., Jiang, L.X., Liu, B.S., Chen, C.T., Fang, D.P., Rao, P.L. and Zhang, M.C. (2014) A Cognitive Perspective on the Safety Communication Factors That Affect Worker Behavior. Journal of Building Construction and Planning Research, 2, 183-197. http://dx.doi.org/10.4236/jbcpr.2014.23017 
Keywords

Communication Style, Worker Cognitive Failure, Unsafe Jobsite Behavior, Construction Safety

\section{Introduction}

Construction safety performance is a global issue that has shown some improvements in recent decades. Workrelated fatality rates in the construction industry decreased by 22\% between 1992 and 2005 and occupational injuries/illnesses with days away from work dropped by $55 \%$ in the same period [1]. Unfortunately, the decline seems to have stopped after 2006, and may actually be reversing from 2011 to 2012 [2]. Moreover, researchers believe that efforts to improve occupational safety by implementing prevailing safety management approaches have stalled and progress has reached a plateau [3]. Research must now focus on improving current safety management paradigms and developing new ones to overcome the current lack of progress.

\subsection{Research Paradigms in Safety Management}

In the realm of construction safety research, three research paradigms have been identified and most studies can be categorized accordingly. The first paradigm is about normative safety, which emphasizes that safe behaviors are defined by the rules and regulations on the jobsite. In other words, behaviors that deviate from the rules and regulations are unsafe. Accordingly, management should emphasize stricter rules and monitoring mechanics to control worker behavior. This logic may work in some industries; however, studies of occupational injury prevention in the construction industry do not seem to support this approach [4]. The studies provide moderate evidence that regulation is not effective in preventing non-fatal and fatal injuries in the construction industry [5]. Moreover, it is not practical or feasible to strictly regulate all working conditions in order to define and monitor unsafe behaviors. A second paradigm focuses on human error, specifically how it occurred and how management should define control measures accordingly. The third paradigm adopts the concept of cognitive engineering to understand why humans make decisions about unsafe behaviors on jobsites. This research focuses on the human choices to explain worker's decisions in complicated but dangerous jobsites. According to the taskcapability model, Mitropoulos, Cupido and Namboodiri [6] focusing on behavior- and cognitive-based safety is the best approach to improve performance by reducing workers' unsafe behaviors in diverse environments.

Since many construction activities involve many project-specific requirements, and the crew members' skills and teamwork practices affect the crew's ability to proceed construction processes, crew coordination and communication are critical to safe performance of construction crews [6]. Many studies have indicated that communication can affect human behaviors through various indicators such as safety culture, or how safety is managed in the workplace, reflecting "the attitudes, beliefs, perceptions and values that employees share in relation to safety" [7]-[13]. These studies outlined the association between communication and safety culture however, the direct relationship between communication and behavior remains blur. According to Vaughan [8] and Mullen [9], human behaviors can be explained by individuals' cognitions. Nonetheless, these associations do not explain how communication affects workers to engage in unsafe behaviors. Comparing the components of safety culture and cognitive failure for unsafe behaviors, common items were clear. For instance, management influence is addressed in safety culture and also captures workers' cognition toward their unsafe behaviors. Therefore, we argue that safety communication affects unsafe behaviors through workers' cognitive failure. The remainder of the introduction describes the current understanding of the relationship between worker/supervisor communication, worker cognition, and unsafe behavior choices in the workplace.

\subsection{Cognition and Unsafe Behaviors}

Mitropoulos [6] explained how workers choose unsafe behaviors with a task-capability model. He suggested that the worker is always balancing capability and workload when performing a specific behavior. Workload can be driven by management requirements, scheduling, work type, etc., while capability is affected by training and other associated background factors. If the workload surpasses the capability, workers tend to perform unsafe behaviors. Similarly, Zhang [10] proposed a cognitive failure model as an explanation for workers' unsafe be- 
haviors. He illustrated that the cognitive reasons for workers' failure to use a safety harness included failure to detect or recognize fall hazards, failure to perceive the need for a safety harness, and failure to use the harness correctly. These failures can occur simultaneously in practice; however, they are illustrated sequentially in Figure 1.

Researchers have stated that hazard detection failure is the main source of unsafe behavior at construction sites. Signal Detection Theory (SDT) originated as a model of perceptual judgment, illustrating how people behave in the premises of detection of a specific type of stimulus. SDT modeled the problems with two types of stimulus, a signal and noise. Theoretically, people detect specific signals without confusion results from nonsignals or noise (other irrelevant stimuli). The correct acceptance of a stimulus as a signal is referred to as a hit. On the other hand, two kinds of noise are considered as the following definitions. A false alarm is the incorrect acceptance of a non-signal; in addition, a miss is the incorrect rejection of a true signal [14]. Abdelhamid [3] correlated workers' risk detection ability and their behaviors with SDT and found a positive correlation between possibility of hazard detection failure and unsafe behavior. Lu [15] and Narang [16] also identified the relationship between signal detection and human behavior with substantial evidence. These researches outlined an interesting research direction, however, to rely solely on this factor as an explanation of unsafe behaviors ignores other potential cognitive factors that may better explain unsafe behaviors.

The term "safety climate" concerns the perceptions of the employees toward the safety of their working environment. Does safety climate explain workers' behaviors? Scholars have debated whether workers' perceptions of the working environment explain their autonomy with regard to choices of unsafe behaviors [11]-[13] [17]. Safety climate indicates the perceptions of the workers, however, the mechanisms by which such perceptions are linked to actual behavior are yet unknown. We argue that individual decision-making mechanics may be a good mediator between communication and unsafe behavior, among other more tangible variables.

\subsection{Communication and Unsafe Behaviors}

Many scholars have explored how jobsite communication affects worker behavior. Kines [18] focused on supervisors' verbal communication with workers and discussed its relationship with safety climate, supporting their argument with statistics on unsafe behaviors. They concluded that higher communication frequency is the key to enhancing safety climate and reducing the frequency of unsafe behaviors at the jobsite. However, they did not clearly explain the relationship between workers' perceptions toward safety and their discretion with regard to unsafe behaviors. Liao [19] analyzed the impact of organizational communication on safety climate using social network analysis. They concluded that patterns of communication networks are interlinked with safety climate,

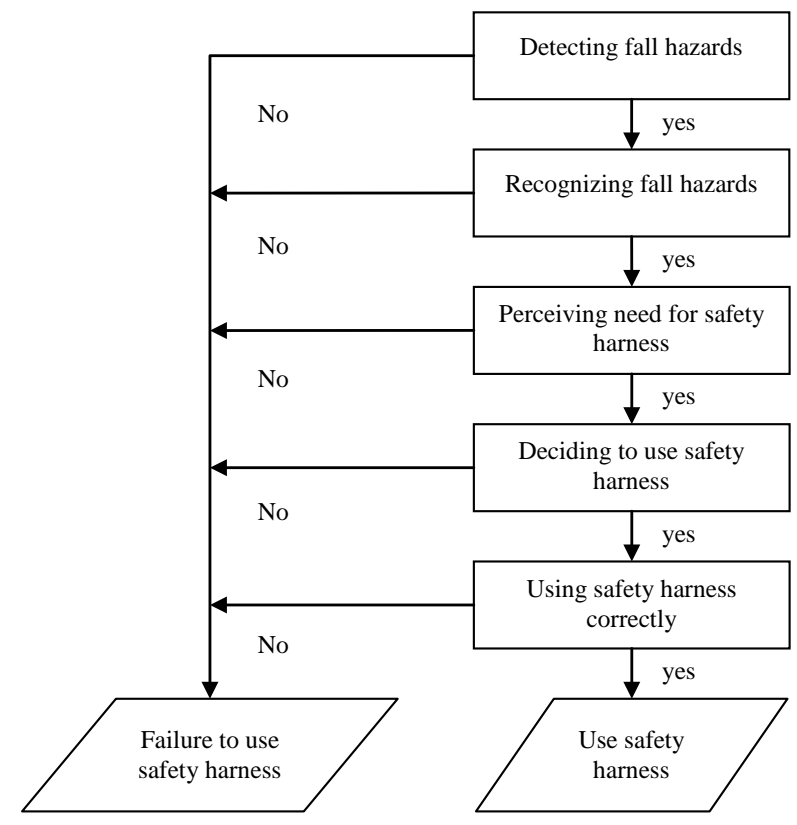

Figure 1. The cognitive failure model (Zhang, 2012). 
which may be related to the occurrence of unsafe behaviors. Unfortunately, they did not include behavioral measurements of communication, suggesting the need to replicate their findings.

\subsection{Communication and Cognition}

Communication channels, strategy, frequency, and quality are the key components of organizational communication [20]. Specifically, communication channels refer to the physical or intangible linkages among message senders and receivers. Kines [18] argued that various verbal communication channels (such as meetings, on-site verbal communication, etc.) exist for most projects; furthermore, the effect of communication on workers' attitudes, and thereby their cognitive process, is stronger when the information is delivered at a higher frequency. Verbal communication was believed to be effective due to the nature of the construction industry as it was more frequently used. However, the quality of the communication can be more important to the ultimate effect of communication [21]-[23]. Engaging in a collaborative communication style, an aspect of communication quality, has a positive influence on treatment outcomes, because it enhances patients' beliefs and therefore their attitudes and cognitive process [24]. We argue that employees' cognition toward safety issues will be strengthened when supervisors engage in positive exchanges with the workers at high frequencies.

Feedback regulates human action through a feedback-standard discrepancy process [25]-[28]. In particular, the receipt of feedback initiates an evaluative process whereby current performance is compared to an objective standard of performance. If a discrepancy is discerned, behavior is hopefully altered in an effort to minimize the discrepancy. Stajkovic and Luthans [29] asserted that "communicated information comes with a guarantee of relevance with cognition" (p. vi). In other words, the message receiver responds to the message sender with a certain level of comprehension about the delivered message, with higher comprehension indicating better quality of delivery. Therefore, in construction, we argue that supervisory feedback can be one of the most powerful incentives influencing job performance.

Various studies also focus on the direction of communication as a means of evaluating its quality. For instance, accident prevention is much more effective when it is based on two-way communication in small groups [30]. Komaki [31] indicated that on-the-job feedback in conjunction with safety training produced a much higher level of compliance than did training alone. Interventions conducted in formal settings such as a workplace and which involved group discussions obtained better effects than did interventions based on one-way communication [32] [33]. Although feedback is largely recognized as a way to potentially improve performance and regulate action by initiating the evaluation of and stimulating the reaction to a mistake [34] [35], there is a lack of detailed explanation on why people react the way they do to feedback [25]-[27] [36].

In summary, the impact of communication on unsafe behaviors requires a more detailed understanding through human cognition, specifically in construction. Therefore, the purpose of this study is to understand the relationship between safety communication and workers' behaviors through a cognitive perspective, so that we can provide rigorous support for jobsite management measures such as safety training.

\section{Methodology}

We first conducted an extensive literature review and identified emerging gaps in existing research (see introduction). The associations among communication, cognitive failure, and unsafe behaviors were interlinked in an input-process-output (I-P-O) model. Standard unsafe behavior checklists were summarized and a typical unsafe behavior was selected according to the results of trial samples. A survey of cognitive failure was developed to include the most frequent unsafe behaviors of steel workers (the rationale of behavior selection is illustrated in the following sections). A checklist for communication behaviors was also developed before our field sampling. An experience sampling approach was adopted to collect data from the selected individuals. Statistical analyses were performed to assess the associations between communication, cognitive failure, and unsafe behaviors. In this study, we consulted four industrial practitioners for development of the checklist of typical unsafe behaviors, questionnaire for interview, and confirmation of research results. The industrial practitioners include three safety managers and a manager of the steel crew from three companies. Their working experience ranges from five to twenty years.

\subsection{The I-P-O Model}

The analytical framework was constructed in an I-P-O model, as shown in Figure 2. The elements are catego- 


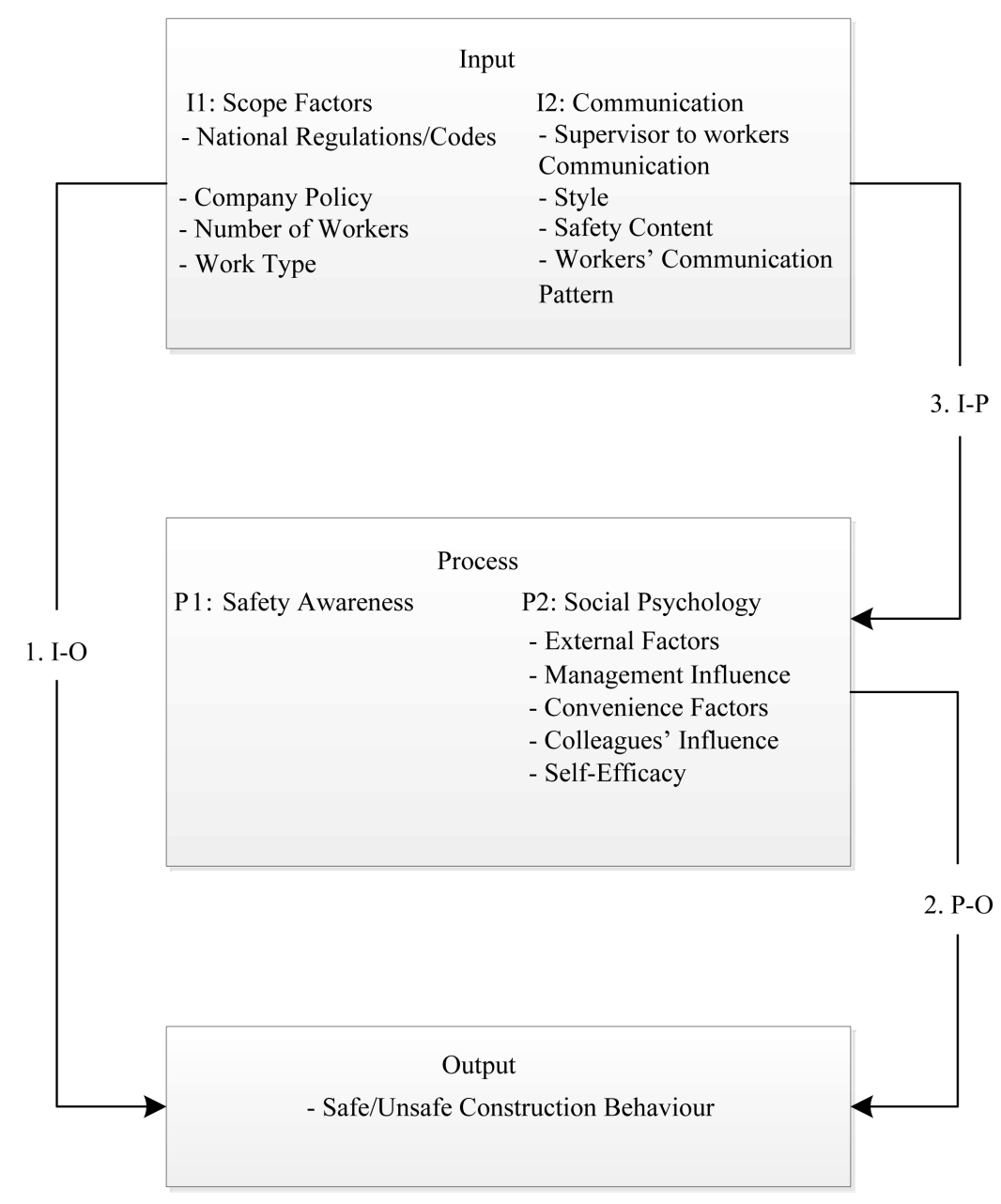

Figure 2. The I-P-O model.

rized in three categories. First was the input category, which included the frequency and quality of supervisors' communication with their crew members. Kines [18] measured communication frequency as the main quality measure with a bivariate variable of whether a conversation included a safety issue. However, this method does not include communication style. Therefore, we incorporated supervisor-worker and worker-worker communication styles into this study. In addition, project type, company policies, regulation codes, crew size, and crew professions were obtained to demonstrate the validity of the associations. Second, this study considered cognitive failure as the workers' "process" toward unsafe behavior. Cognitive failure, in this instance, included situational awareness (i.e., risk detection of the workers) and a range of socio-psychological factors. Specifically, we measured risk detection in terms of SDT, while other cognitive variables (such as perceiving needs for safety measures) were captured by the questions used in Zhang's study [37] The output category includes simply safe/unsafe behaviors. Safe/unsafe behaviors were determined by a pre-defined checklist developed from literature review and input from two safety managers employed by the general contractor and subcontractor.

\subsection{On-Site Observations}

\subsubsection{Behaviors}

We selected steel worker behaviors for this study because these workers tend to have a relatively simple production process that could be observed from a distance. According to Zhang [10], unsafe behaviors can be categorized as actively creating safety hazards (ex. throwing a hammer) and passively conduct self-protection (ex. walking on the safety paths). Five typical unsafe behaviors were selected and listed in the checklist for sampling the workers' behaviors. The checklist was validated by field professionals as behaviors with the highest possi- 
bility of occurrence when workers are at work. We patrolled the jobsite and recorded worker behavior twice per day (10 am and $3 \mathrm{pm}$ ) because previous studies considered these times the high peaks of unsafe behavior [38].

It was not feasible to examine cognitive failure for all of the identified unsafe behaviors because of the workers' tight schedules. Based on Zhang's [10] finding, we assumed the mechanics of cognitive failure are similar regarding the same behavior across all of the steel workers, we identified a "representative behavior" and examined the mechanics of cognitive failure for that behavior. In this case, the representative behavior was determined by identifying which of the five typical unsafe behaviors occurred more than $30 \%$ of the observed samples. Then, we discussed the behaviors with a safety manager of the general contractors, and a safety manager and the crew leader of the subcontractors. We chose "walking on the safety paths" to be the subject of the interview because all workers are likely to travel around the worksite for different task demands, and travelling around the worksite may take a large percentage of time. Therefore, there was reason to believe workers would be more familiar with the safety behavior when questions were asked. We then expanded the communication and cognitive failure questionnaire for further examination.

\subsubsection{Cognitive Failure}

According to Zhang's study, workers' failure to use safety harnesses can result from poor hazard detection, lack of hazard recognition, lack of the perceived need for the safety harness, decision to not use the safety harness, and incorrect usage of the safety harness. Hazard detection was measured using SDT in this study. The theory can be used to detect workers' situational awareness by portraying the same question regarding safety in a number of different ways. The worker's risk identification ability was tested by the examiners (who were trained for cross-checking prior to field test in order to reduce observers' bias) with Figure 3 and two questions listed below.

\section{A Sample Question:}

It is absolutely safe for you to walk around the jobsite like this.

Answer:

o (1) Absolutely Safe

o (2)

o (3) Injurious

0 (4)

o (5) Fatal

How Confident are you on your answer?

o (1) Completely Uncertain

o (2)

o (3) Moderate Confidence

o (4)

o (5) Absolutely Confident

The workers' situational awareness was measured by their responses. In this study, a worker had either a conservative or a risky awareness regarding the safety situation at work. A Likert scale was used to record workers' responses to the above questions, which ranged from safe to unsafe and uncertain to certain. The workers' certainty was expressed in terms of percentages ( $0 \%$ to $100 \%)$. To determine the boundary between

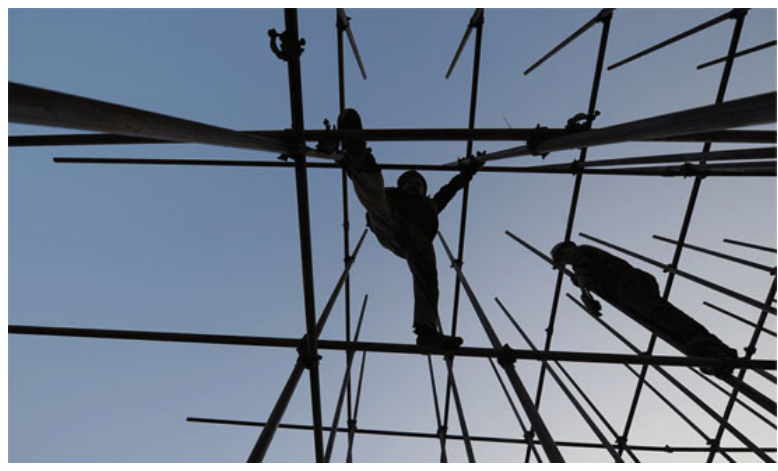

Figure 3. A sample picture of SDT test. 
"conservative" and "risky" strategies, we calculated the $\beta$ value of each worker and compared it to the optimum $\beta$ value (which will be explained in the later part of this paragraph), and this value remained constant according to set scenarios designed by the researchers [15] [39]. In this study, the optimum value was 1 , as there were 4 basic questions and photos reflecting two scenarios: working on elevated paths and using safety paths. The question and photos of each scenario were set out evenly to assess workers' awareness of those situations. The workers' responses were then compared with the optimum value; result values less than the optimum value were considered a "conservative strategy" and values greater than the optimum value were seen as workers adopting a "risky strategy" [15] [16] [40]. In this study, for a specific construction behavior, a safe and an unsafe scenario were addressed in two separate sets of questions assessed by each worker. Each worker assessed each scenario with regard to how safe the behavior is and how confident the worker is about his assessment. In this study, the optimum $\beta$ value is 1 because there is only one question addressed in safe and unsafe behavior. For example, if a worker's response to the first question was 2 and the response to the second was 4 , the individual $\beta$ value defined by $\mathrm{Lu}$ [39] can be calculated as 0.5 . Since 0.5 (individual $\beta$ value) is less than 1 (optimum $\beta$ value), the situation awareness of such a worker is classified as "risky", indicating that the worker tends to fail to detect safety risk on jobsites.

For other social psychology factors, a total of thirteen questions were asked in the survey (Table 1). The survey was conducted by two examiners to one examinee each time and a cross-checking mechanism was implemented during the survey. If one of the examiners disagreed with the other, the same question was asked again by the other examiner to reduce worker response bias. The questions were categorized into five main factors, including convenience factors, managerial impact, external factors, influence of other workers, and self-efficacy, and an average score of each factor was produced for further analysis.

\subsubsection{Communication}

In this study, we defined communication quality in terms of its content and style. Communication content was evaluated as whether safety was mentioned in the interview with the participants. For instance, if, during the interview, a worker mentioned any safety issue regarding personal protective equipment (PPE) in the latest conversation with crew lead or any colleague, the respondent was denoted as "mentioned safety", otherwise, the respondent was marked with "did not mention safety". For communication style, we categorized three subgroups: coaching approach [41] [42], abusive approach [43]-[45] and experience-sharing or storytelling [46]. Coaching approach uses the perspectives of teaching and advising repetitively to improve your safety awareness [47]. Abusive communication approach was identified to acknowledge safety practices by shouting, swearing, insulting, and punishing workers [48] [49]. Storytelling or experience-sharing approach is to gain trust between the superior-worker relationships into personal levels [46] which uses personal experience and reasoning for

Table 1. The social psychology survey for unsafe behaviors.

\begin{tabular}{|c|c|c|c|c|c|c|}
\hline & Questions & $\begin{array}{l}\text { Strongly } \\
\text { agree }\end{array}$ & Agree & Neutral & Disagree & $\begin{array}{l}\text { Strongly } \\
\text { disagree }\end{array}$ \\
\hline 1 & It will be much safer when walking on safety paths at work. & & & & & \\
\hline 2 & Travelling on safety paths will decrease my productivity. & & & & & \\
\hline 3 & The main contractor requires me to walk on safety paths at all times. & & & & & \\
\hline 4 & It is too far for me to travel on safety paths. & & & & & \\
\hline 5 & There are no safety walk paths on-site. & & & & & \\
\hline 6 & My supervisor will think I'm slow when I travel on safety paths. & & & & & \\
\hline 7 & My supervisor will remind me to walk on safety paths when I'm not. & & & & & \\
\hline 8 & My colleague will remind me to walk on safety paths when I'm not. & & & & & \\
\hline 9 & My colleagues will think I'm slow when I travel on safety paths. & & & & & \\
\hline 10 & My colleagues will admire me if I disregard travelling on safety walk paths. & & & & & \\
\hline 11 & I know which ones are the safety walk paths. & & & & & \\
\hline 12 & I will travel on safety walk paths next time I work. & & & & & \\
\hline 13 & I will normally travel on safety walk paths. & & & & & \\
\hline
\end{tabular}


correct practices. The experience sampling method (ESM) was adopted to collect data for this part of the study. Interviewees were asked to recall the most recent conversation with their crew leader and their colleagues about safety issues. The content was recorded, analyzed, and coded in terms of the following: communication content, communication style, the setting (meeting/training program, on-site/at work, privately), who participated in the conversation, and if there was any feedback during the conversation.

In order to assess the validity of this study, we intentionally collected data from two steel crews at the same jobsite managed by the same general contractor and another crew at a second jobsite managed by another general contractor. We intentionally selected two Chinese sate-owned enterprises and find the training profiles and demographic information of the crews are similar for better control of the workers' skill and knowledge in comparisons. The patterns were then compared between the three groups. A total of 142 participants (three crew leaders and 139 steel workers) were surveyed and data was collected in the communication and cognition portion of the study. Further, the data were recorded anonymously without other personal information. We obtained 2046 behavior samples from job sampling, and each behavior sample can be observed from the same worker.

\section{Analyses and Results}

The data were analyzed according to the I-P-O model described above. The factors of the input group (communication frequency, quality, and originator) were first analyzed alone with the output (unsafe behaviors). The process factors (cognitive-failure-related factors) were compared against the output as the second phase of analysis. Third, the input factors were then correlated with process factors in order to analyze their relationships. During each step, we first compared the results in general, then broke the data into groups and checked whether the results retained the pattern. Finally, the results of the abovementioned three steps were discussed altogether and explanations as well as interpretations were made accordingly. Data were analyzed by ANOVA, T-test, or chisquare analyses with SPSS ${ }^{\circledR}$.

\subsection{Input-Output Relationship}

Again, the first analyses focused on the relationship between communication factors and safety behavior (Tables 2-5).

Table 2. Verbal communication (with supervisor) by group.

\begin{tabular}{lcccc}
\hline Verbal communication & Group 1 $(\mathrm{N}=53)$ & Group 2 $(\mathrm{N}=41)$ & Group 3 (N = 48) & All $(\mathrm{N}=142)$ \\
\hline Safety content & $98.1 \%$ & $97.6 \%$ & $97.9 \%$ & $97.9 \%$ \\
Feedback & $7.5 \%$ & $2.4 \%$ & $14.6 \%$ & $8.5 \%$ \\
\hline
\end{tabular}

Table 3. Communication styles by group.

\begin{tabular}{|c|c|c|c|c|}
\hline Supervisor's communication style & Group $1(\mathrm{~N}=53)$ & Group $2(\mathrm{~N}=41)$ & Group $3(\mathrm{~N}=48)$ & All $(\mathrm{N}=142)$ \\
\hline Coaching & $15.1 \%$ & $12.2 \%$ & $50.0 \%$ & $26.1 \%$ \\
\hline Storytelling or experience-sharing & $62.3 \%$ & $39.0 \%$ & $41.7 \%$ & $48.6 \%$ \\
\hline Abusive & $22.6 \%$ & $48.8 \%$ & $8.3 \%$ & $25.4 \%$ \\
\hline
\end{tabular}

Table 4. Verbal communication (with colleague) by group.

\begin{tabular}{|c|c|c|c|c|}
\hline Verbal communication (with colleague) & Group $1(\mathrm{~N}=53)$ & Group $2(\mathrm{~N}=41)$ & Group $3(\mathrm{~N}=48)$ & All $(\mathrm{N}=142)$ \\
\hline Safety content & $0.0 \%$ & $0.0 \%$ & $6.3 \%$ & $2.1 \%$ \\
\hline Feedback & $0.0 \%$ & $0.0 \%$ & $6.3 \%$ & $2.1 \%$ \\
\hline
\end{tabular}

Table 5. Safety behaviors by group.

\begin{tabular}{lcccc}
\hline Safe/unsafe construction behavior & Group 1 (3003) & Group 2 (1078) & Group 3 (804) & All (4885) \\
\hline Safe & $63.9 \%$ & $75.6 \%$ & $90.4 \%$ & $75.7 \%$ \\
Unsafe & $36.1 \%$ & $24.4 \%$ & $9.6 \%$ & $24.3 \%$ \\
\hline
\end{tabular}


Overall, more than $97 \%$ of the samples referred to safety issues. There was no distinguishable difference among the groups, meaning that the pattern was consistent across the groups and projects. Nonetheless, only 8.5\% of the sample included feedback from the conversations between the workers and crew leaders. The average feedback at Group 3 was approximately 10\% higher than that at Group 1 and Group 2. These results regarding feedback revealed that workers mostly followed the instructions of crew leaders without any response.

In terms of communication style, it seems storytelling or experience-sharing (demonstrating a specific behavior in a safe manner) was the most prevalent safety management approach, making up close to $50 \%$ of the communication in total. Coaching (repeatedly reminding workers of a specific behavior) and abusive comprised the other 50\%. The crew leader at Group 3 had a distinguishable difference in awareness usage from the lead at Group 1 and Group 2, but there was relatively little difference in storytelling or experience-sharing. This meant that in addition to frequently demonstrating how to perform safety measures, the crew leaders at Group 3 repeated reminders about safety on the jobsite. Compared to the groups at Group 3, crew leaders at Group 1 and Group 2 used a more abusive communication style. We found that the crew leaders using this kind of approach were often swearing at their workers, who often complained about this fact throughout our sampling procedure.

We examined the communication among the workers, finding almost no communication feedback among the workers. Among all the groups, the workers at Group 3 had a higher percentage than did the other crews, who reported no feedback; however, the percentage was still relatively low. The content normally covered safety helmets, safety harnesses, and reminders about work at the edge. The feedback regarding the workers on the jobsite was low as well.

Overall, the observations revealed that about $24.3 \%$ of the behaviors witnessed were unsafe, which is relatively lower than the 36\% found in previous studies [50] [51]. The unsafe behavior in Group 3 was found to be approximately $15 \%$ less than that of Group 1 and $27 \%$ less than that of Group 2.

More unsafe behaviors were found at Group 1 and Group 2 than at Group 3. Despite a high percentage of verbal communication addressing safety issues, no difference was distinguishable among the observed groups. The crew leader of Group 3 communicated using more repeated verbal reminders and relatively fewer demonstrations of the safety measures to guide workers. Feedback at Group 1 and Group 2 was higher than Group 3 for both lead-worker and worker-worker communication. It seems better communication quality in terms of both style and feedback related to a lower percentage of unsafe behavior.

According to the analyses above, Group 2 had fewer unsafe behaviors than Group 1. The two groups at site 1 may conflict because the two groups were surveyed and sampled in different phases of the project. Group 1 was surveyed during foundation work on the project, when safety protection systems were not available and we considered more behavior unsafe. When Group 2 was surveyed at a later phase of the project, more safety protection systems were installed (fall arrest nets, scaffolding barriers, safety paths) and therefore worker behaviors were safer.

\subsection{Process-Output Relationship}

Secondly, the relationship between worker cognition and safety behavior was evaluated (Tables 6-8).

Approximately $76 \%$ of the workers displayed conservative situational awareness at their jobsite. Even with four-fifths of the sample workers considered able to detect risks, a remarkable difference was observed between the jobsites. The workers at Group 3 perceived hazards more conservatively than did those at Group 1 and Group 2. This finding may correlate with workers' attitude toward unsafe behaviors. The more the negative attitude towards unsafe behavior, the lower the opportunity for workers to detect safety hazards.

The groups also differed significantly in terms of the socio-psychological factors. The lower the factor score, the higher probability that a worker encountered cognitive failure. Average scores were higher than 3.5 for all factors except self-efficacy, indicating that the workers had a moderate tendency to encounter cognitive failure. Across crews, workers did not have significantly different perceptions of external conditions (see Table 8). For

Table 6. Analysis of situational awareness by group.

\begin{tabular}{|c|c|c|c|c|}
\hline Situational awareness & Group $1(\mathrm{~N}=53)$ & Group $2(\mathrm{~N}=41)$ & Group $3(\mathrm{~N}=48)$ & All $(\mathrm{N}=142)$ \\
\hline Conservative & $66.0 \%$ & $73.2 \%$ & $89.6 \%$ & $76.1 \%$ \\
\hline Risky & $34.0 \%$ & $26.8 \%$ & $10.4 \%$ & $23.9 \%$ \\
\hline
\end{tabular}


Table 7. Descriptive statistics of socio-psychological factors.

\begin{tabular}{lcccccccc}
\hline Socio-psychological factors & $\begin{array}{c}\text { Group 1 } \\
(\mathrm{N}=53)\end{array}$ & $\mathrm{SD}$ & $\begin{array}{c}\text { Group 2 } \\
(\mathrm{N}=41)\end{array}$ & $\mathrm{SD}$ & $\begin{array}{c}\text { Group 3 } \\
(\mathrm{N}=48)\end{array}$ & $\mathrm{SD}$ & $\begin{array}{c}\text { All } \\
(\mathrm{N}=142)\end{array}$ & SD \\
\hline External factors & 3.7 & 0.9 & 3.5 & 0.9 & 3.8 & 0.9 & 3.7 & 0.9 \\
Convenience factors & 3.2 & 0.6 & 4.0 & 0.6 & 3.5 & 0.7 & 3.5 & 0.7 \\
Management influences & 3.7 & 0.4 & 3.3 & 0.5 & 3.8 & 0.6 & 3.6 & 0.5 \\
Colleague influences & 4.0 & 0.5 & 2.6 & 0.4 & 4.1 & 0.6 & 3.6 & 0.8 \\
Self-efficacy & 3.0 & 0.7 & 2.5 & 0.9 & 3.8 & 0.9 & 3.0 & 0.9 \\
\hline
\end{tabular}

Table 8. ANOVA test of socio-psychological factors by group.

\begin{tabular}{lcccccc}
\hline $\begin{array}{l}\text { Social psychology } \\
\text { (between groups comparison) }\end{array}$ & \multicolumn{3}{c}{ Tukey HSD } & F & $P$ \\
\cline { 2 - 5 } & Group 1 (N = 53) & Group 2 (N = 41) & Group 3 (N=48) & 3.8125 & 1.624 & 0.201 \\
\hline External factors & 3.6604 & 3.4634 & 3.5317 & 15.384 & 0.000 \\
Convenience factors & 3.2075 & $3.9756^{*}$ & $3.7937^{*}$ & 9.726 & 0.000 \\
Management influences & $3.7208^{*}$ & 3.3390 & $4.0958^{*}$ & 117.143 & 0.000 \\
Colleague influences & $4.0208^{*}$ & 2.5927 & $3.7292^{*}$ & 22.0393 & 0.000
\end{tabular}

*Represents the highest Tukey HSD score where they are significant.

management influences, colleague influences, and self-efficacy, the average score at Group 3 and Group 1 is significantly higher than that of Group 2, indicating that the workers at these groups encountered less cognitive failure than those at Group 2. In terms of convenience factors, compared to Group 1, workers in Group 2 tended to believe that walking on the safety route did not jeopardize their work, tended to have better ability to identify safer routes, and followed instructions to use the safety route. This is probably because of workers' generic attitude toward unsafe behaviors is different. As shown in Table 5, highest percentage of safe behaviors were sampled at Group 3, consistent with the lowest likelihood of cognitive failure at the same group as shown in Table 7. According to Zhang [10], socio-psychological factors do have direct/indirect relationship with behavior intension which is a significant indicator to unsafe behaviors. Even the attitude of the groups may differ the patterns observed with regard to management influence, colleague influence, and self-efficacy, the behavior patterns are positively correlate with that of cognitive failure.

\subsection{Input-Process Relationship}

The third step was to evaluate the relationship between communication and worker cognition. T-test and Chisquare analysis were performed to examine the relationship between communication and cognitive failure (Tables 9-11).

Neither safety content nor communication style correlated significantly with situational awareness. No significant relationship was found among the groups. This result echoes Stanton's [52] and Walker's [53] research that situational awareness cannot be altered by short-term management measures but may possibly be affected by long-term background factors such as education, philanthropy issues, etc.

Similarly, the results indicated in Table 10 show that communication frequency did not correlate with cognitive failure except for the management influences factor. The higher the frequency of safety communication, the less influence the crew leader had on the jobsite. This interesting finding probably arose when reminders were mostly provided in meetings rather than during construction activities. Given this finding, perhaps trainings for foreman should encourage the reminder made during construction activities, including trainings.

In general, workers felt more comfortable walking on the safety route with repeated reminders from the crew leader. Repeated reminders made the workers more aware of and able to identify the safety route, and more willing to use it. Such a trend was further extended to between group comparisons. Specifically, storytelling or experience-sharing style was further correlated with these factors. The trend of Group 1, Group 2 and Group 3 
Table 9. Communication style and safety content with situational awareness.

\begin{tabular}{|c|c|c|c|c|c|c|}
\hline Relationship & Site & & Statistical analysis & Results & $P$ & $\mathrm{~F}$ \\
\hline \multirow{8}{*}{$\mathrm{I}-\mathrm{P}$} & \multirow[b]{2}{*}{$\begin{array}{c}\text { All } \\
(\mathrm{N}=142)\end{array}$} & $\begin{array}{l}\text { Communication style } \\
\text { and SA }\end{array}$ & Chi-square & $X^{2}=0.955, \mathrm{df}=2$ & 0.62 & \\
\hline & & $\begin{array}{l}\text { Safety content } \\
\text { (supervisor) and SA }\end{array}$ & T-test & $\begin{array}{l}\text { Conservative: } \mathrm{N}=114 \text {, } \\
\text { Mean = 0.6631, SD = 0.29574 } \\
\text { Risky: } \mathrm{N}=28, \text { Mean = 0.7196, } \\
\mathrm{SD}=0.32167\end{array}$ & 0.278 & 1.185 \\
\hline & \multirow[b]{2}{*}{$\begin{array}{l}\text { Group } 1 \\
(\mathrm{~N}=53)\end{array}$} & $\begin{array}{l}\text { Communication style } \\
\text { and SA }\end{array}$ & Chi-square & $X^{2}=0.121, \mathrm{~N}=94, \mathrm{df}=2$ & 0.941 & \\
\hline & & $\begin{array}{l}\text { Safety content } \\
\text { (supervisor) and SA }\end{array}$ & T-test & $\begin{array}{l}\text { Conservative: } \mathrm{N}=69 \text {, } \\
\text { Mean = 0.6539, SD = 0.29130 } \\
\text { Risky: } \mathrm{N}=25, \text { Mean }=0.7060 \text {, } \\
\mathrm{SD}=0.32810\end{array}$ & 0.145 & 2.155 \\
\hline & \multirow[b]{2}{*}{$\begin{array}{l}\text { Group } 2 \\
(\mathrm{~N}=41)\end{array}$} & $\begin{array}{l}\text { Communication style } \\
\text { and SA }\end{array}$ & Chi-square & $X^{2}=7.966, \mathrm{~N}=53, \mathrm{df}=3$ & 0.047 & \\
\hline & & $\begin{array}{l}\text { Safety content } \\
\text { (supervisor) and SA }\end{array}$ & T-test & $\begin{array}{l}\text { Conservative: } \mathrm{N}=39 \\
\text { Mean = } 0.6231, \mathrm{SD}=0.27854 \\
\text { Risky: } \mathrm{N}=14, \text { Mean }=0.7736 \text {, } \\
\mathrm{SD}=0.33754\end{array}$ & 0.306 & 1.069 \\
\hline & \multirow[b]{2}{*}{$\begin{array}{l}\text { Group } 3 \\
(\mathrm{~N}=48)\end{array}$} & $\begin{array}{l}\text { Communication style } \\
\text { and SA }\end{array}$ & Chi-square & $X^{2}=3.2, \mathrm{~N}=48, \mathrm{df}=2$ & 0.202 & \\
\hline & & $\begin{array}{l}\text { Safety content } \\
\text { (supervisor) and SA }\end{array}$ & T-test & $\begin{array}{l}\text { Conservative: } \mathrm{N}=45 \\
\text { Mean }=0.6771, \mathrm{SD}=0.30520 \\
\text { Risky: } \mathrm{N}=3, \text { Mean }=0.8333 \text {, } \\
\mathrm{SD}=0.28868\end{array}$ & 0.256 & 1.324 \\
\hline
\end{tabular}

Table 10. Correlations between communication frequency and socio-psychological factors.

\begin{tabular}{|c|c|c|c|c|c|c|c|c|}
\hline \multirow{3}{*}{ Correlations } & \multicolumn{8}{|c|}{ Safety content (supervisor) } \\
\hline & \multicolumn{2}{|c|}{ Group $1(\mathrm{~N}=53)$} & \multicolumn{2}{|c|}{ Group $2(\mathrm{~N}=41)$} & \multicolumn{2}{|c|}{ Group $3(\mathrm{~N}=48)$} & \multicolumn{2}{|c|}{ All $(\mathrm{N}=142)$} \\
\hline & $r$ & $P$ & $r$ & $P$ & $r$ & $P$ & $r$ & $P$ \\
\hline External factors & -0.1 & 0.7 & -0.1 & 0.6 & 0.2 & 0.1 & 0.0 & 0.8 \\
\hline Convenience factors & 0.0 & 0.9 & 0.0 & 0.8 & -0.1 & 0.6 & 0.0 & 0.9 \\
\hline Management influences & 0.2 & 0.2 & -0.5 & $0.0^{*}$ & -0.2 & 0.2 & -0.1 & 0.1 \\
\hline Colleague influences & 0.1 & 0.4 & 0.0 & 0.9 & 0.1 & 0.4 & 0.1 & 0.5 \\
\hline Self-efficacy & 0.0 & 0.9 & 0.3 & 0.1 & 0.1 & 0.5 & 0.1 & 0.2 \\
\hline
\end{tabular}

All $r$ values are Pearson's correlation coefficients. All P-values are 2-tailed.

Table 11. Correlations between communication styles and socio-psychological factors.

\begin{tabular}{cccccccccc}
\hline \multirow{2}{*}{ ANOVA } & \multicolumn{9}{c}{ Communication style } \\
\cline { 2 - 9 } & \multicolumn{1}{c}{ Group 1 $(\mathrm{N}=53)$} & \multicolumn{2}{c}{ Group 2 $(\mathrm{N}=41)$} & \multicolumn{2}{c}{ Group 3 (N = 48) } & \multicolumn{2}{c}{ All $(\mathrm{N}=142)$} \\
\cline { 2 - 9 }$y$ & $\mathrm{~F}$ & $P$ & $\mathrm{~F}$ & $P$ & $\mathrm{~F}$ & $P$ & $\mathrm{~F}$ & $P$ \\
\hline External factors & 0.9 & 0.4 & 0.7 & 0.5 & 0.0 & 1.0 & 2.2 & 0.1 \\
Convenience factors & $21.6^{\mathrm{A}}$ & $0.0^{*}$ & 1.1 & 0.3 & 3.5 & $0.0^{*}$ & $5.4^{\mathrm{A}}$ & $0.0^{*}$ \\
Management influence & $10.0^{\mathrm{AC}}$ & $0.0^{*}$ & 0.3 & 0.7 & $3.7^{\mathrm{AC}}$ & $0.0^{*}$ & $13.3^{\mathrm{A}}$ & $0.0^{*}$ \\
Colleague influences & 0.0 & 1.0 & 0.2 & 0.9 & 1.3 & 0.3 & $4.2^{\mathrm{A}}$ & $0.0^{*}$ \\
Self-efficacy & $10.5^{\mathrm{A}}$ & $0.0^{*}$ & 2.1 & 0.1 & $3.1^{\mathrm{AC}}$ & 0.1 & $24.9^{\mathrm{A}}$ & $0.0^{*}$ \\
\hline
\end{tabular}

"Means significant result; ${ }^{\mathrm{A}}$ Means the score of awareness significantly higher than that of the other communication style(s); ${ }^{\mathrm{C}}$ Means the score of cognition significantly higher than that of the other communication style(s).

are quite similar in that both high coaching style and storytelling or experience-sharing style correlated with less cognitive failure.

In summary, repeated reminders were found to be the most useful strategy for the workers. Although storytelling or experience-sharing style (demonstrating the safe behaviors) should lead to less cognitive failure compared to abusive strategies, no significant effect was found for situational awareness. Therefore, this strategy 
should be used at the onset of training or to rectify workers' behaviors. Coaching style (repeated measures) should be continued across the process of the project.

\subsection{I-P-0 Relationship}

Finally, the entire model (I-P-O) was reviewed. From the analyses performed above, communication does affect unsafe behaviors through cognitive failure. Specifically, repeated reminders were found to be the most effective way to reduce the probability of cognitive failure, thus leading to less unsafe behavior. Repeated reminders of safe behaviors correlated with management influence such that the workers remained aware that managers prioritized safety highly. Repeated reminders also guided the workers to identify the safety route. Although storytelling or experience-sharing style may potentially correlate with the workers' perception regarding the priority of the project objectives, managers' influence, and self-efficacy, the data overall did not demonstrate significant results on these factors. Demonstrating safe behaviors is necessary but not sufficient. Repeated reminders can be more effective in managing safe behaviors during projects at jobsites in China. Despite this finding, we suggest that demonstrating safe behaviors to workers is an important communication style. Frequently, leaders remind workers to perform safe behaviors with the inaccurate assumption that all workers know how to perform the behaviors.

Another finding of this research is that the frequency of safe communication embedded in the conversations did not correlate with either situational awareness or the studied socio-psychological factors. This is likely because safe communication has been addressed frequently enough to be considered normal conversation. Therefore, communication frequency should be considered a main variable affecting cognitive failures linked to behavior. From a cognitive failure perspective, one should conclude that communication quality is a very important variable affecting unsafe behavior, given enough communication frequency.

\section{Discussions}

Although some conclusions can be drawn, the results of this study should be interpreted cautiously. First, as we employed convenience sampling, the sample size is relatively small and we had to make assumptions about potential homogeneity of the sample. Additionally, the feedback among workers was too low to perform any correlation analysis with other variables in the process and output categories. Since we believe that greater worker communication would be helpful for workers' cognitions and thus lead to fewer unsafe behaviors, this should be evaluated in future studies. Management should be aware that worker feedback occurred much less than expected and contractors and subcontractors should strengthen worker's feedback to one another in ultra-hazardous tasks.

Crew turnover may also interfere with safe behaviors and communication. In this study, we inferred that crew turnover may be a key factor affecting communication quality and thus resulting in greater cognitive failure and more unsafe behaviors. Turnover can also lead to unstable relationships between the crew leader and crew, thus leading to increased cognitive failure. If this is the case, the impact of communication on cognitive failure can be blurred and a longitudinal study on specific crews working on the same job site could be used to evaluate it.

\section{Conclusions}

This study aimed to understand the mechanics of how communication factors affect unsafe workplace behavior though human cognition. We collected behavioral data on the workers through observation and collected data on communication style and cognitive failure via an experience sampling approach. We concluded that although communication frequency is important to safety performance, it is not as significant as communication quality. Crew leaders should remind workers about performing safe behaviors by showing them how to do it. This would lead to fewer workers experiencing less cognitive failure because they will become convinced that they can perform their work safely, which will lead to less unsafe behavior. The results of this study bridge the gaps of previous research, providing an insight of the association between group communication and individual cognitive issues regarding unsafe behaviors at construction jobsites.

Future research may need to focus on the following topics. For instance, the present study sheds light on the relationship between communication and unsafe behavior through human cognition. However, the role of safety culture in this relationship is yet unclear. There may be an interesting association between culture and human 
cognition which can be modified by communication frequency and styles, indirectly affecting human behavior. Such a topic is worth investigation. Another interesting question is whether human cognitions about safety reduce productivity. This issue needs to be examined to strengthen current arguments supporting modification of human cognition for safer behavior.

\section{Acknowledgements}

This study is funded by the United Technology Center. The project number is 20133000265 . We would specifically thank our interviewees Quinn Li, Yuhang Sun, Zhen Dong, and Weiwei Li.

\section{References}

[1] Dong, X., et al. (2008) The Construction Chart Book: The US Construction Industry and Its Workers.

[2] Bonde, J.P.E. (2008) Psychosocial Factors at Work and Risk of Depression: A Systematic Review of the Epidemiological Evidence. Occupational and Environmental Medicine, 65, 438-445. http://dx.doi.org/10.1136/oem.2007.038430

[3] Abdelhamid, T.S. and Everett, J.G. (2000) Identifying Root Causes of Construction Accidents. Journal of Construction Engineering and Management, 126, 52-60. http://dx.doi.org/10.1061/(ASCE)0733-9364(2000)126:1(52)

[4] Lehtola, M.M., van der Molen, H.F., Lappalainen, J., Hoonakker, P.L.T., Hsiao, H., Haslam, R.A., Hale, A.R. and Verbeek, J.H. (2008) The Effectiveness of Interventions for Preventing Injuries in the Construction Industry: A Systematic Review. American Journal of Preventive Medicine, 35, 77-85. http://dx.doi.org/10.1016/j.amepre.2008.03.030

[5] Spangenberg, S. (2009) An Injury Risk Model for Large Construction Projects. Risk Management, 11, 111-134. http://dx.doi.org/10.1057/rm.2009.5

[6] Mitropoulos, P., Cupido, G. and Namboodiri, M. (2009) Cognitive Approach to Construction Safety: Task DemandCapability Model. Journal of Construction Engineering and Management, 135, 881-889. http://dx.doi.org/10.1061/(ASCE)CO.1943-7862.0000060

[7] Cox, B. (1991) Cox on Cox: An English Curriculum for the 1990s. Hodder \& Stoughton, London.

[8] Vaughan, E. (1993) Chronic Exposure to an Environmental Hazard: Risk Perceptions and Self-Protective Behavior. Health Psychology, 12, 74. http://dx.doi.org/10.1037/0278-6133.12.1.74

[9] Mullen, J. (2004) Investigating Factors That Influence Individual Safety Behavior at Work. Journal of Safety Research, 35, 275-285. http://dx.doi.org/10.1016/j.jsr.2004.03.011

[10] Zhang M. (2012) Cognitive Mechanism of Construction Worker's Unsafe Behaviors and its Application. Tsinghua University, Beijing.

[11] Atkin, B. and Borgbrant, J. (2007) Proceedings of 4th Nordic Conference on Construction Economics and Organization. Development Processes in Construction Management.

[12] Cooper, M.D. and Phillips, R.A. (2004) Exploratory Analysis of the Safety Climate and Safety Behavior Relationship. Journal of Safety Research, 35, 497-512. http://dx.doi.org/10.1016/j.jsr.2004.08.004

[13] DeJoy, D.M. (2005) Behavior Change versus Culture Change: Divergent Approaches to Managing Workplace Safety. Safety Science, 43, 105-129. http://dx.doi.org/10.1016/j.ssci.2005.02.001

[14] Swets, J.A. (1996) Signal Detection Theory and ROC Analysis in Psychology and Diagnostics: Collected Papers. Lawrence Erlbaum Associates, Mahwah, N.J.

[15] Lu, Y., Hinze, J. and Li, Q. (2011) Developing Fuzzy Signal Detection Theory for Workers’ Hazard Perception Measures on Subway Operations. Safety Science, 49, 491-497. http://dx.doi.org/10.1016/j.ssci.2010.11.008

[16] Narang, P. and Abdelhamid, T. (2006) Quantifying Workers’ Hazard Identification Ability Using Fuzzy Signal Detection Theory. Annual Conference of the International Group for Lean Construction (IGLC-14), Santiago, 25-27 July 2006, 497-509.

[17] Swuste, P., Frijters, A. and Guldenmund, F. (2012) Is It Possible to Influence Safety in the Building Sector? A Literature Review Extending from 1980 until the Present. Safety Science, 50, 1333-1343. http://dx.doi.org/10.1016/j.ssci.2011.12.036

[18] Kines, P., Andersen, L.P., Spangenberg, S., Mikkelsen, K.L, Dyreborg, J. and Zohar, D. (2010) Improving Construction Site Safety through Leader-Based Verbal Safety Communication. Journal of Safety Research, 41, 399-406. http://dx.doi.org/10.1016/j.jsr.2010.06.005

[19] Liao, P.C., Thomas, S.R. and O’Brien, W.J. (2013) Measuring Information Dependency for Construction Engineering 
Projects. Journal of Civil Engineering and Management, 19, 177-183. http://dx.doi.org/10.3846/13923730.2012.743924

[20] O’Reilly, C.A. (1982) Variations in Decision Makers’ Use of Information Sources: The Impact of Quality and Accessibility of Information. Academy of Management Journal, 25, 756-771. http://dx.doi.org/10.2307/256097

[21] Daft, R.L. and Lengel, R.H. (1986) Organizational Information Requirements, Media Richness and Structural Design. Management Science, 32, 554-571. http://dx.doi.org/10.1287/mnsc.32.5.554

[22] Stohl, C. and Redding, W.C. (1987) Messages and Message Exchange Processes.

[23] Mohr, J.J. and Sohi, R.S. (1996) Communication Flows in Distribution Channels: Impact on Assessments of Communication Quality and Satisfaction. Journal of Retailing, 71, 393-415. http://dx.doi.org/10.1016/0022-4359(95)90020-9

[24] Bultman, D.C. and Svarstad, B.L. (2000) Effects of Physician Communication Style on Client Medication Beliefs and Adherence with Antidepressant Treatment. Patient Education and Counseling, 40, 173-185. http://dx.doi.org/10.1016/S0738-3991(99)00083-X

[25] Carver, C.S. (1979) A Cybernetic Model of Self-Attention Processes. Journal of Personality and Social Psychology, 37, 1251.

[26] Hollenbeck, J.R., Williams, C.R. and Klein, H.J. (1989) An Empirical Examination of the Antecedents of Commitment to Difficult Goals. Journal of Applied Psychology, 74, 18-23. http://dx.doi.org/10.1037/0021-9010.74.1.18

[27] Bandura, A. and Cervone, D. (1986) Differential Engagement of Self-Reactive Influences in Cognitive Motivation. Organizational Behavior and Human Decision Processes, 38, 92-113. http://dx.doi.org/10.1016/0749-5978(86)90028-2

[28] Locke, E.A. and Latham, G.P. (1990) A Theory of Goal Setting \& Task Performance. Prentice-Hall, Inc., Upper Saddle River.

[29] Stajkovic, A.D. and Luthans, F. (2003) Behavioral Management and Task Performance in Organizations: Conceptual Background, Meta-Analysis, and Test of Alternative Models. Personnel Psychology, 56, 155-194. http://dx.doi.org/10.1111/j.1744-6570.2003.tb00147.x

[30] Ajzen, I. (2002) Perceived Behavioral Control, Self-Efficacy, Locus of Control, and the Theory of Planned Behavior. Journal of Applied Social Psychology, 32, 665-683. http://dx.doi.org/10.1111/j.1559-1816.2002.tb00236.x

[31] Ajzen, I. and Fishbein, M. (1980) Understanding Attitudes and Predicting Social Behaviour.

[32] Geller, E.S. and Hahn, H.A. (1984) Promoting Safety Belt Use at Industrial Sites: An Effective Program for Blue Collar Employees. Professional Psychology: Research and Practice, 15, 553-564. http://dx.doi.org/10.1037/0735-7028.15.4.553

[33] Dunton, S., Perkins, D.D. and Zopf, K.J. (1990) The Impact of Worksite-Based Health Risk Appraisal Programs on Observed Safety Belt Use. Health Education Research, 5, 207-216. http://dx.doi.org/10.1093/her/5.2.207

[34] Kopelman, R.E. (1986) Objective Feedback. Generalizing from Laboratory to Field Settings, 119, 145.

[35] Kluger, A.N. and DeNisi, A. (1996) The Effects of Feedback Interventions on Performance: A Historical Review, a Meta-Analysis, and a Preliminary Feedback Intervention Theory. Psychological Bulletin, 119, 254-284. http://dx.doi.org/10.1037/0033-2909.119.2.254

[36] Ashford, S.J. and Cummings, L.L. (1983) Feedback as an Individual Resource: Personal Strategies of Creating Information. Organizational Behavior and Human Performance, 32, 370-398. http://dx.doi.org/10.1016/0030-5073(83)90156-3

[37] Zhang, M. and Fang, D. (2012) A Continuous Behavior-Based Safety Strategy for Persistent Safety Improvement in Construction Industry. Automation in Construction.

[38] Fang, D.P., Huang, X.Y., Xin, C. and Hinze, J. (2005) Safety Management in Construction. Waterpower Press, Beijing.

[39] Lu, Y., Li, Q. and Hinze, J. (2011) Subway System Safety Risk Analysis Based on Bayesian Network. In: Wu, D.D., Ed., Modeling Risk Management in Sustainable Construction, Springer, Berlin, 219-227. http://dx.doi.org/10.1007/978-3-642-15243-6_25

[40] Abdelhamid, T., Patel, B., Howell, G. and Mitropoulos, P. (2003) Signal Detection Theory: Enabling Work near the Edge. Annual Conference on Lean Construction, Blacksburg, 22-24 July 2003, 243-256.

[41] Bakhtari, H. (1995) Cultural Effects on Management Style: A Comparative Study of American and Middle Eastern Management Styles. International Studies of Management and Organization, 25, 97-97.

[42] Cigularov, K.P., Chen, P.Y. and Rosecrance, J. (2010) The Effects of Error Management Climate and Safety Communication on Safety: A Multi-Level Study.

[43] Yoo, S. and Lee, S.M. (1987) Management Style and Practice of Korean Chaebols. California Management Review, 29, 
95-110. http://dx.doi.org/10.2307/41162133

[44] Kassing, J.W. and Avtgis, T.A. (1999) Examining the Relationship between Organizational Dissent and Aggressive Communication. Management Communication Quarterly, 13, 100-115. http://dx.doi.org/10.1177/0893318999131004

[45] Thau, S., Bennett, R.J., Mitchell, M.S. and Marrs, M.B. (2009) How Management Style Moderates the Relationship between Abusive Supervision and Workplace Deviance: An Uncertainty Management Theory Perspective. Organizational Behavior and Human Decision Processes, 108, 79-92. http://dx.doi.org/10.1016/j.obhdp.2008.06.003

[46] Gill, R. (2011) Using Storytelling to Maintain Employee Loyalty during Change. International Journal of Business \& Social Science, 2, 23-32.

[47] Humphreys, J.H. (2002) Transformational Leader Behavior, Proximity and Successful Services Marketing. Journal of Services Marketing, 16, 487-502. http://dx.doi.org/10.1108/08876040210443373

[48] Keashly, L. (1997) Emotional Abuse in the Workplace: Conceptual and Empirical Issues. Journal of Emotional Abuse, 1, 85-117. http://dx.doi.org/10.1300/J135v01n01_05

[49] Mitchell, M.S. and Ambrose, M.L. (2007) Abusive Supervision and Workplace Deviance and the Moderating Effects of Negative Reciprocity Beliefs. Journal of Applied Psychology, 92, 1159-1168. http://dx.doi.org/10.1037/0021-9010.92.4.1159

[50] Williams, J.H. and Geller, E.S. (2000) Behavior-Based Intervention for Occupational Safety: Critical Impact of Social Comparison Feedback. Journal of Safety Research, 31, 135-142. http://dx.doi.org/10.1016/S0022-4375(00)00030-X

[51] Howell, G.A., Ballard, G., Abdelhamid, T.S. and Mitropoulos, P. (2002) Working near the Edge: A New Approach to Construction Safety. Annual Conference on Lean Construction, 49-60.

[52] Stanton, N.A., Chambers, P. and Piggott, J. (2001) Situational Awareness and Safety. Safety Science, 39, 189-204. http://dx.doi.org/10.1016/S0925-7535(01)00010-8

[53] Walker, G.H., Stanton, N.A., Kazi, T.A., Salmon, P.M. and Jenkins, D.P. (2009) Does Advanced Driver Training Improve Situational Awareness? Applied Ergonomics, 40, 678-687. http://dx.doi.org/10.1016/j.apergo.2008.06.002 
Scientific Research Publishing (SCIRP) is one of the largest Open Access journal publishers. It is currently publishing more than 200 open access, online, peer-reviewed journals covering a wide range of academic disciplines. SCIRP serves the worldwide academic communities and contributes to the progress and application of science with its publication.

Other selected journals from SCIRP are listed as below. Submit your manuscript to us via either submit@scirp.org or Online Submission Portal.
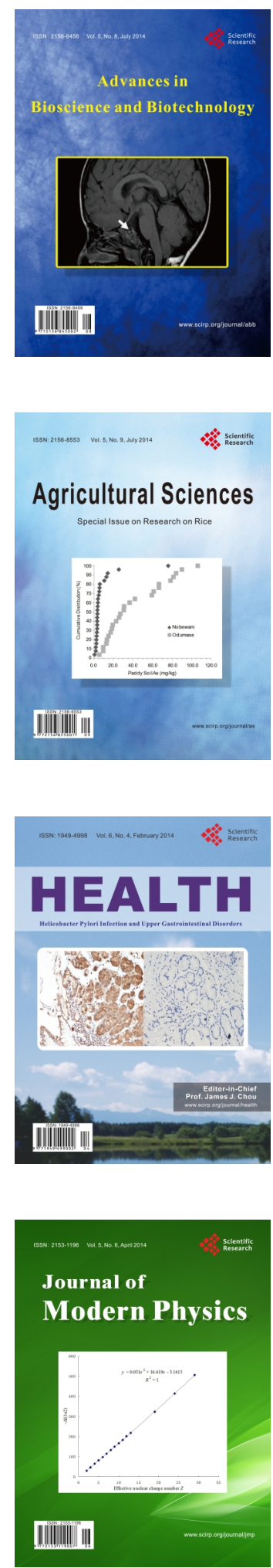
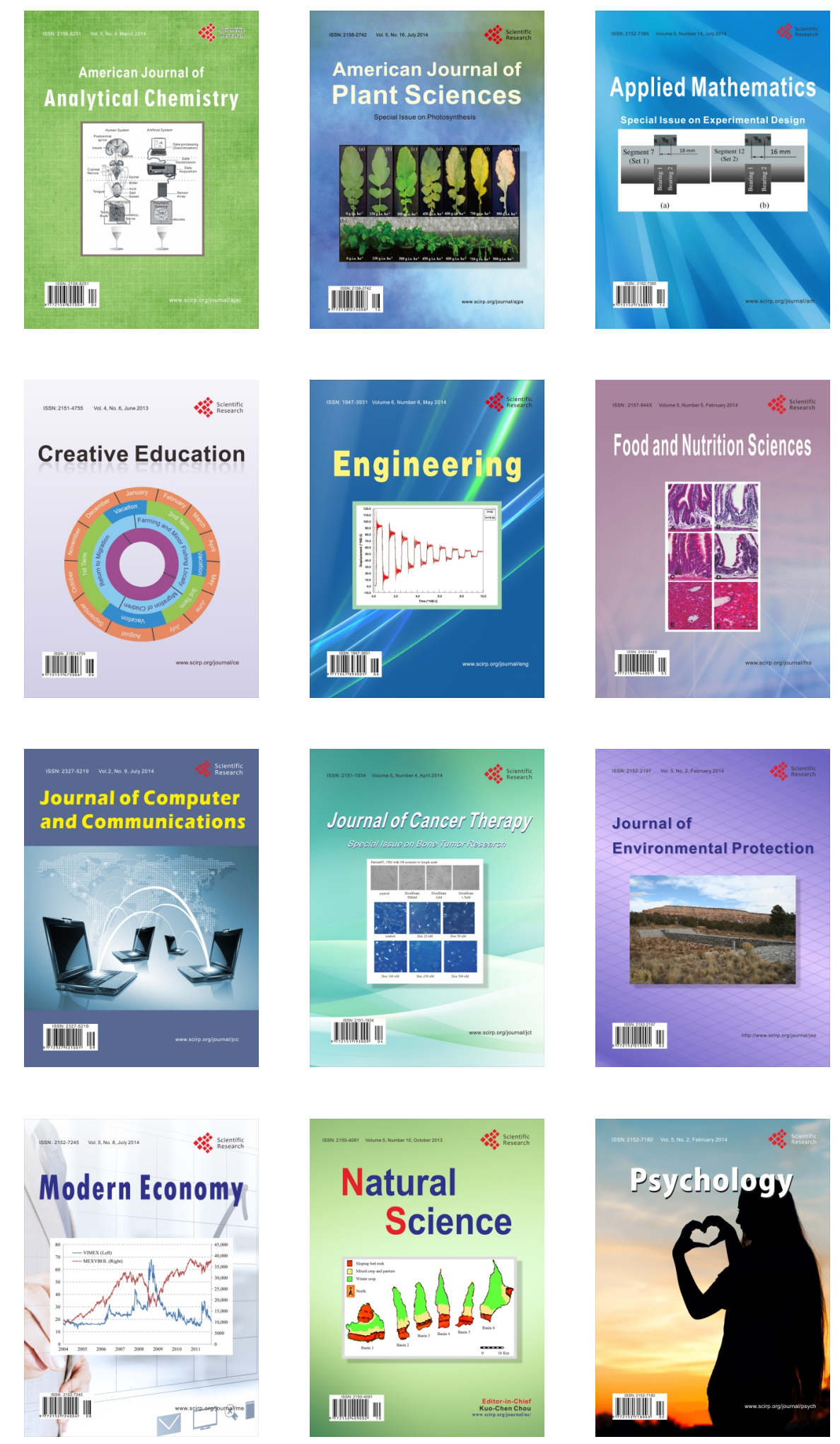